Proof. For $i=1,2, \cdots n,(x+n-i) \equiv(y+n-i) \quad(\bmod t k)$ so that $(x+n-1)(x+n-2) \cdots(x) \equiv(y+n-1)(y+n-2) \cdots(y) \quad(\bmod t k)$. Also from the definition of $t,(n !, t k)=t$ since $t$ is the product of highest powers of $p_{1}, p_{2}, \cdots p_{q}$ which are contained in $n$ !. So,

$$
f_{n, x}=\frac{(x+n-1) \cdots(x)}{n !} \equiv \frac{(y+n-1) \cdots(y)}{n !}=f_{n, y}(\bmod k)
$$

which proves the theorem.

It is not asserted in the theorem that $t k$ is the smallest period. In fact, easy examples show that in many cases a value for $t$ can be found which is strictly smaller than the one specified in the theorem. According to Mathematical Reviews (v. 20, 1959, Review no. 1653), the smallest period has evidently been found by S. Zabek [5] to be $t k$ where

$$
t={p_{1}}^{\alpha_{1}}{p_{2}}^{\alpha_{2}} \cdots p_{q}^{\alpha_{q}} \text {. }
$$

This information may be quite useful in numerically searching for solutions to (5) since these congruences limit the number of solutions that could possibly exist, thereby reducing the amount of machine time needed for the search.

The University of Colorado

Boulder, Colorado

1. J. C. P. Miller, \& M. F. C. Woollett, "Solutions of the Diophantine equation $x^{3}+$ $y^{3}+z^{3}=k$," London Math. Soc., v. 30, 1955, p. 101-110.

2. L. Dickson, History of the Theory of Numbers, v. 2, Chap. 1. (Reprint: Stechert, New York, 1934).

3. S. CHOwla and others, "The Diophantine equation $x^{3}+y^{3}+z^{3}=x+y+z$," submitted to Norske Vid. Selsk. Forh. Trondheim.

4. S. Segal, "A note on pyramidal numbers," American Math. Monthly, (in press).

5. Swiatomir Zabex, "Sur la periodicite modulo $m$ des suites de nombres $\left(\begin{array}{l}n \\ k\end{array}\right)$, , Ann. Univ. Mariae Curi-Sklodowska, Sect. A, 10 (1956), p. 37-47.

\title{
Note on Osculatory Rational Interpolation $\dagger$
}

\section{By Herbert E. Salzer}

Abstract. In $n$-point osculatory interpolation of order $r_{i}-1$ at points $x_{i}$, $i=1,2, \cdots, n$, by a rational expression $N(x) / D(x)$, where $N(x)$ and $D(x)$ are polynomials $\sum a_{j} x^{j}$ and $\sum b_{j} x^{j}$, we use the lemma that the system (1) $\left\{N\left(x_{i}\right) / D\left(x_{i}\right)\right\}^{(m)}=f^{(m)}\left(x_{i}\right), m=0,1, \cdots, r_{i}-1$, is equivalent to (2) $N^{(m)}\left(x_{i}\right)=$ $\left\{f\left(x_{i}\right) D\left(x_{i}\right)\right\}^{(m)}, m=0,1, \cdots, r_{i}-1, D\left(x_{i}\right) \neq 0$. This equivalence does not require $N(x)$ or $D(x)$ to be a polynomial or even a linear combination of given functions. The lemma implies that (1), superficially non-linear in $a_{j}$ and $b_{j}$, being the same as (2), is actually linear. For the $n$-point interpolation problem, the linear system, of order $\sum_{i=1}^{n} r_{i}$, which might be large, is replaceable by separate linear

Received April 20, 1961.

$\dagger$ Much of the material in the present note is contained in an entirely independent (still unpublished) study by Henry C. Thacher, Jr., who was kind enough to send the writer a copy of his preliminary draft. 
systems of orders $r_{i}$ (or even $r_{i}+r_{i+1}+\cdots+r_{i+j}$ when conveniently small) by applying the lemma to the continued fraction (3) $N(x) / D(x)=a_{1,0}+\frac{x-x_{1} \mid}{\mid a_{1,1}}+$ $\frac{x-x_{1} \mid}{\mid a_{1,2}}+\cdots+\frac{x-x_{1} \mid}{\mid a_{1, r_{1}-1}}+\frac{x-x_{1} \mid}{\mid a_{2,0}}+\frac{x-x_{2} \mid}{\mid a_{2,1}}+\cdots+\frac{x-x_{2} \mid}{\mid a_{2, r_{2}-1}}+\frac{x-x_{2} \mid}{\mid a_{3,0}}+\cdots$ $+\frac{x-x_{n-1} \mid}{\mid a_{n, 0}}+\frac{x-x_{n} \mid}{\mid a_{n, 1}}+\cdots+\frac{x-x_{n} \mid}{\mid a_{n, r_{n}-1}}$. In (3), which has the property (proven in two ways) that the determination of $a_{i, m}$ is independent of all $a$ 's that follow, we find $a_{i, m}$ stepwise, but several at a time (instead of singly which is more tedious), retrieving them readily from the solutions of those lower-order linear systems.

1. Introduction and General Lemma. In $n$-point osculatory rational interpolation for a given function $f(x)$ by $N(x) / D(x)$, where $N(x)=\sum a_{j} x^{j}$ and $D(x)=$ $\sum b_{j} x^{j}$, we have to find the coefficients $a_{j}$ and $b_{j}$ from the following $d=\sum_{i=1}^{n} r_{i}$ conditions:

$$
d^{m}\{N(x) / D(x)\} /\left.d x^{m}\right|_{x=x_{i}}=f^{(m)}\left(x_{i}\right), \quad \begin{aligned}
m & =0,1, \cdots, r_{i}-1, \\
i & =1,2, \cdots, n .
\end{aligned}
$$

The $N(x)$ and $D(x)$ are usually taken to be of nearly equal degree, i.e., for odd $d$, both of degree [d/2], and for even $d$, of degree [d/2] and [d/2] -1 respectively.

One answer to (1) is provided by Thiele's reciprocal difference formula for confluent arguments [1]. But that requires the build-up and tabulation of a reciprocal difference scheme involving confluent forms, which might be too cumbersome to handle for a large total number of conditions. The present approach considers a more direct solution of (1), and avoids confluent reciprocal differences.

Whenever in (1) some $r_{i}>1$, the determination of $a_{j}$ and $b_{j}$ after differentiation of the left member, appears offhand to involve the solution of a non-linear system of equations. In reality, (1) may always be solved by an equivalent linear system. Before citing the general lemma which establishes this equivalence, it is instructive to verify the first few cases. Let $N$ and $D$ denote any functions of $x$, not necessarily linear combinations, the subscript $i$ denote the argument $x_{i}$, and $D_{i} \neq 0$.

Ordinary rational interpolation, $(N / D)_{i}=f_{i}$, is, of course, equivalent to $N_{i}=$ $(D f)_{i}$.

First-order osculatory interpolation,

$$
(N / D)_{i}=f_{i}, \quad(N / D)_{i}{ }^{\prime}=f_{i}{ }^{\prime},
$$

may be expressed as $N_{i}=(D f)_{i}$ and $N_{i}{ }^{\prime} / D_{i}-N_{i} D_{i}{ }^{\prime} / D_{i}{ }^{2}=f_{i}{ }^{\prime}$. In this last equation, replace $N_{i} / D_{i}$ by $f_{i}$ and multiply by $D_{i}$, so that (2) is replaced by the equivalent

$$
N_{i}=(D f)_{i}, \quad N_{i}{ }^{\prime}=(D f)_{i}{ }^{\prime} .
$$

Whenever $N$ and $D$ are linear combinations of specified functions, the system $\left(2^{\prime}\right)$ is linear in the coefficients.

Similarly, for second-order osculatory interpolation, namely,

$$
(N / D)_{i}=f_{i}, \quad(N / D)_{i}{ }^{\prime}=f_{i}{ }^{\prime}, \quad(N / D)_{i}{ }^{\prime \prime}=f_{i}{ }^{\prime \prime},
$$

carrying out the differentiation in the last equation of $(3)$, in view of the equivalence 
of the first two equations in (3) to $\left(2^{\prime}\right)$, we find (3) to be equivalent to the system

$$
N_{i}=(D f)_{i}, \quad N_{i}^{\prime}=(D f)_{i}^{\prime}, \quad N_{i}^{\prime \prime}=(D f)_{i}{ }^{\prime \prime} .
$$

The foregoing equivalence relations suggest this general lemma for any $N$ and $D$, as long as $D_{i} \neq 0$ : The system

$$
(N / D)_{i}^{(m)}=f_{i}^{(m)}, \quad m=0,1, \cdots, r
$$

is equivalent to

$$
N_{i}^{(m)}=(D f)_{i}^{(m)}, \quad m=0,1, \cdots, r .
$$

The proof is immediate by induction. Assume the equivalence of (4) and (4') up to order $r-1$. Then first assume $\left(4^{\prime}\right)$, and apply Leibnitz's theorem to both $\left(4^{\prime}\right)$ just for $m=r$ and to $N_{i}^{(r)}=\{(N / D) D\}_{i}^{(r)}$. In the former, replace $f_{i}^{(m)}$ by $(N / D)_{i}^{(m)}$, $m=0,1, \cdots, r-1$. Comparison of terms shows that $(N / D)_{i}^{(r)}=f_{i}^{(r)}$, so that $\left(4^{\prime}\right)$ implies all of (4). Conversely, starting from (4) and applying it to the Leibnitz formula for $N_{i}^{(r)}=\{(N / D) D\}_{i}^{(r)}$, we obtain the equation in $\left(4^{\prime}\right)$ for $m=r$, which establishes all of $\left(4^{\prime}\right)$.

A discrete case analogue of this lemma (i.e., for finite differences or divided differences, instead of derivatives) is established even more readily. Thus the equality of the differences of $N / D$ and $f$ at $x_{i}$ implies that $(N / D)_{j}=f_{j}$ at every $x_{j}$ of the total $\sum_{i=1}^{n} r_{i}$ points (assume now that every $D_{j} \neq 0$ ), from which follows the equality of $N_{j}$ and $(D f)_{j}$ which, in turn, implies the equality of the differences of $N$ and $D f$ at every $x_{i}$. The lemma above is seen to be a limiting confluent case of the finite difference analogue.

Application of the lemma to (1) reduces the general problem of osculatory rational interpolation to the solution of just a linear system for the $a_{j}$ and $b_{j}$, which is thus not harder than the problem of osculatory polynomial interpolation. It is apparent from the inductive process in the proof of the lemma that (unlike the polynomial case) we could not expect to obtain a linear system from any modification of (1) where $m$ either fails to start at 0 or to run consecutively, even at just a single point $x_{i}$.

By this lemma, even when $N(x)$ and $D(x)$ are not polynomials, but linear combinations of preassigned known functions $\psi_{j}(x)$ in place of $x^{j}$, we may replace the system (1) by the equivalent linear system

$$
N^{(m)}\left(x_{i}\right)=d^{m}\{D(x) f(x)\} /\left.d x^{m}\right|_{x-x_{i}}, \quad \begin{aligned}
m & =0,1, \cdots, r_{i}-1, \\
i & =1,2, \cdots, n,
\end{aligned}
$$

where every $D\left(x_{i}\right) \neq 0$.

2. Application of Lemma to Continued Fraction Interpolation. From now on we shall consider just the most important case when $N(x)$ and $D(x)$ are polynomials. The lemma may be applied to solve (1) or $\left(1^{\prime}\right)$ by a number of linear systems, each of much lower order than that of $\left(1^{\prime}\right)$, namely, $\sum_{i=1}^{n} r_{i}$, which might be inconveniently large, by the method described below.

We may express $N(x) / D(x)$ in (1) as the continued fraction 


$$
\begin{aligned}
N(x) / D(x)= & a_{1,0}+\frac{x-x_{1} \mid}{\mid a_{1,1}}+\frac{x-x_{1} \mid}{\mid a_{1,2}}+\cdots+\frac{x-x_{1} \mid}{\mid a_{1, r_{1}-1}}+\frac{x-x_{1} \mid}{\mid a_{2,0}} \\
+\frac{x-x_{2} \mid}{\mid a_{2,1}}+\cdots+ & \frac{x-x_{2} \mid}{\mid a_{2, r_{2}-1}}+\frac{x-x_{2} \mid}{\mid a_{3,0}}+\cdots \\
& +\frac{x-x_{n-1} \mid}{\mid a_{n, 0}}+\frac{x-x_{n} \mid}{\mid a_{n, 1}}+\cdots+\frac{x-x_{n} \mid}{\mid a_{n, r_{n-1}}}
\end{aligned}
$$

Now (5) has the useful property that each coefficient $a_{i, m}$ which may be found from (1), involves just the preceding coefficients $a_{j, k}$ and is completely independent of every following $a_{j, k}$. This property may be seen from the limiting confluent form of Thiele's continued fraction formula in terms of reciprocal differences [1]. Another direct way to establish this property without employing the Thiele reciprocal difference scheme, is by induction upon (5). Thus, at any $x_{i}$ in (1) and (5), the interpolating equation for the next higher order derivative, say the $m$ th, may be shown to involve $a_{i, m}$ as the only new quantity, provided that the same is true for all coefficients preceding $a_{i, m}$, likewise down to $a_{i, r_{i}-1}$, then $a_{i+1,0}$ is found from the value of $f\left(x_{i+1}\right)$, and so on down to $a_{n, r_{n}-1}$. By inspection, this property holds for the first few coefficients $a_{1,0}, a_{1,1}$, which suffices to complete the induction.

To apply this property, we recall the procedure in the special case of (5) when every $r_{i}=1$ (i.e., ordinary rational interpolation) and each $a_{i, 0}$ is found from

$$
f\left(x_{i}\right)=\frac{p_{i}\left(x_{i}\right)}{q_{i}\left(x_{i}\right)}=\frac{a_{i, 0} p_{i-1}\left(x_{i}\right)+\left(x_{i}-x_{i-1}\right) p_{i-2}\left(x_{i}\right)}{a_{i, 0} q_{i-1}\left(x_{i}\right)+\left(x_{i}-x_{i-1}\right) q_{i-2}\left(x_{i}\right)},
$$

where $p_{i}(x)$ and $q_{i}(x)$ denote the numerator and denominator of the $i$ th convergent [2]. (We may differ here from some standard notation by referring to $a_{1,0}$ in (5) as the first, instead of the zero-th convergent.)

The same idea as in (6) can be used for the stepwise determination of the entire block of $r_{i}$ coefficients $a_{i, 0}, a_{i, 1}, \cdots, a_{i, r_{i}-1}$ simultaneously. Assuming that from knowledge of $a_{1,0}, a_{1,1}, \cdots, a_{i-1, r_{i-1}-1}$ we have found in (5) the convergents $p_{s-1}(x) / q_{s-1}(x), p_{s-2}(x) / q_{s-2}(x)$, where $s-1=\sum_{j=1}^{i-1} r_{j}$. Then

$$
N(x) / D(x)=\frac{R_{i}(x) p_{s-1}(x)+\left(x-x_{i-1}\right) p_{s-2}(x)}{R_{i}(x) q_{s-1}(x)+\left(x-x_{i-1}\right) q_{s-2}(x)}
$$

where

$$
\begin{aligned}
R_{i}(x)=a_{i, 0}+\frac{x-x_{i} \mid}{\mid a_{i, 1}} & +\frac{x-x_{i} \mid}{\mid a_{i, 2}}+\cdots \\
& +\frac{x-x_{i} \mid}{\mid a_{i, r_{i}-1}}+\frac{x-x_{i} \mid}{\mid a_{i+1,0}}+\frac{x-x_{i+1} \mid}{\mid a_{i+1,1}}+\cdots+\frac{x-x_{n} \mid}{\mid a_{n, r_{n}-1}}
\end{aligned}
$$

We may neglect that part of $R_{i}(x)$ which proceeds from $\frac{x-x_{i} \mid}{\mid a_{i+1,0}}+\cdots$ in determining $a_{i, 0}, a_{i, 1}, \cdots, a_{i, r_{i}-1}$ from the $r_{i}$ osculatory interpolating conditions on (7) at $x=x_{i}$. Denoting that truncated $R_{i}(x)$ by $R_{i}{ }^{*}(x)=S_{i}(x) / T_{i}(x)$, we apply the general lemma at $x=x_{i}$, finding first the coefficients of the polynomials $S_{i}(x)$ and $T_{i}(x)$ to satisfy 


$$
\begin{aligned}
& \left.\left\{S_{i}(x) p_{s-1}(x)+\left(x-x_{i-1}\right) T_{i}(x) p_{s-2}(x)\right\}^{(m)}\right|_{x=x_{i}} \\
& =\left.\left[f(x)\left\{S_{i}(x) q_{s-1}(x)+\left(x-x_{i-1}\right) T_{i}(x) q_{s-2}(x)\right\}\right]^{(m)}\right|_{x=x_{i}}, \\
& \quad m=0,1, \cdots, r_{i}-1 .
\end{aligned}
$$

The next operation is to retrieve the coefficients $a_{i, 0}, \cdots, a_{i, r_{i}-1}$ from those of $S_{i}(x)$ and $T_{i}(x)$. Finally, the convergents to $(5), p_{s}(x), q_{s}(x), p_{s+1}(x), q_{s+1}(x), \cdots$ are found by the usual recurrence scheme

$$
\begin{array}{ll}
p_{s+t}(x)=a_{i, t} p_{s+t-1}(x)+\begin{array}{cl}
\left(x-x_{i-1}\right) \\
\left(x-x_{i}\right)
\end{array} p_{s+t-2}(x), & \text { for } t=0 \\
q_{s+t}(x)=a_{i, t} q_{s+t-1}(x)+\begin{array}{ll}
\left(x-x_{i-1}\right) \\
\left(x-x_{i}\right)
\end{array} q_{s+t-2}(x), & \text { for } t=0, \\
\text { for } t=1(1) r_{i}-1
\end{array}
$$

The cycle of (9), retrieval of coefficients $a_{i, m}$ and (10), $\left(10^{\prime}\right)$ is repeated, but with $i$ replaced by $i+1$ and $s$ replaced by $s+r_{i}$, etc. For a succession of smaller values of $r_{i}$, say $r_{i}=2, r_{i+1}=3, \cdots$ it may be convenient to apply (9) at several points at once.

This procedure, which involves the solution of a number of separate linear systems of orders $r_{i}$ (or $r_{i}+r_{i+1}+\cdots+r_{i+j}$ when fairly small), is an intermediate one between that of solving for all $\sum_{i=1}^{n} r_{i}$ coefficients of $N(x) / D(x)$ at one time, where the linear system may be inconveniently large, and that of solving for each

\begin{tabular}{|c|c|c|}
\hline$r_{i}$ & $S_{i}(x)$ & $T_{i}(x)$ \\
\hline 1 & $a_{0}$ & 1 \\
\hline 2 & $a_{1} a_{0}+\left(x-x_{i}\right)$ & $a_{1}$ \\
\hline 3 & $a_{2} a_{1} a_{0}+\left(a_{2}+a_{0}\right)\left(x-x_{i}\right)$ & $a_{2} a_{1}+\left(x-x_{i}\right)$ \\
\hline 4 & $\begin{array}{r}a_{3} a_{2} a_{1} a_{0}+\left(a_{3} a_{2}+a_{3} a_{0}+a_{1} a_{0}\right)\left(x-x_{i}\right) \\
+\left(x-x_{i}\right)^{2}\end{array}$ & $a_{3} a_{2} a_{1}+\left(a_{3}+a_{1}\right)\left(x-x_{i}\right)$ \\
\hline 5 & $\begin{array}{l}a_{4} a_{3} a_{2} a_{1} a_{0}+\left(a_{4} a_{3} a_{2}+a_{4} a_{3} a_{0}\right. \\
\left.\quad+a_{4} a_{1} a_{0}+a_{2} a_{1} a_{0}\right)\left(x-x_{i}\right) \\
\quad+\left(a_{4}+a_{2}+a_{0}\right)\left(x-x_{i}\right)^{2}\end{array}$ & $\begin{array}{l}a_{4} a_{3} a_{2} a_{1}+\left(a_{4} a_{3}+a_{4} a_{1}\right. \\
\left.+a_{2} a_{1}\right)\left(x-x_{i}\right)+\left(x-x_{i}\right)^{2}\end{array}$ \\
\hline 6 & $\begin{array}{l}a_{5} a_{4} a_{3} a_{2} a_{1} a_{0}+\left(a_{5} a_{4} a_{3} a_{2}+a_{5} a_{4} a_{3} a_{0}\right. \\
\left.\quad+a_{5} a_{4} a_{1} a_{0}+a_{5} a_{2} a_{1} a_{0}+a_{3} a_{2} a_{1} a_{0}\right)(x \\
\left.\quad-x_{i}\right)+\left(a_{5} a_{4}+a_{5} a_{2}+a_{5} a_{0}+a_{3} a_{2}\right. \\
\left.\quad+a_{3} a_{0}+a_{1} a_{0}\right)\left(x-x_{i}\right)^{2}+\left(x-x_{i}\right)^{3}\end{array}$ & $\begin{array}{l}a_{5} a_{4} a_{3} a_{2} a_{1}+\left(a_{5} a_{4} a_{3}+a_{5} a_{4} a_{1}\right. \\
\left.+a_{5} a_{2} a_{1}+a_{3} a_{2} a_{1}\right)\left(x-x_{i}\right) \\
+\left(a_{5}+a_{3}+a_{1}\right)\left(x-x_{i}\right)^{2}\end{array}$ \\
\hline
\end{tabular}
separate coefficient $a_{i, m}$ in (5), from an equation like the following, when $m>0$,

$$
f^{(m)}\left(x_{i}\right)=\left.\left\{\frac{a_{i, m} p_{r}(x)+\left(x-x_{i}\right) p_{r-1}(x)}{a_{i, m} q_{r}(x)+\left(x-x_{i}\right) q_{r-1}(x)}\right\}^{(m)}\right|_{x=x_{i}}, \quad r=\sum_{j=1}^{i-1} r_{j}+m,
$$

SCHedule I: $S_{i}(x) / T_{i}(x)$ 
(remembering now that we must retain the denominator in the right member of (11) because it is for a single $m$ ) and finding $N(x)$ and $D(x)$ from (10), $\left(10^{\prime}\right)$. Since there would be $\sum_{i=1}^{n} r_{i}$ such equations (11), that is likely to involve more work than the present scheme.

In using (9) at just a single point $x_{i}, S_{i}(x)$ and $T_{i}(x)$ are kept in terms of $\left(x-x_{i}\right)^{j}$, whereas $p_{s-1}(x), q_{s-1}(x), p_{s-2}(x)$ and $q_{s-2}(x)$ are in terms of $x^{j}$. After retrieving the coefficients $a_{i, m}$, it is natural to obtain $p_{s+t}(x), q_{s+t}(x)$ from (10), $\left(10^{\prime}\right)$ in terms of $x^{j}$, then $S_{i+1}(x)$ and $T_{i+1}(x)$ are found as polynomials in $x-x_{i+1}$, etc.

For the convenience of the user we list in Schedule I the explicit expressions for $S_{i}(x)$ and $T_{i}(x)$ as polynomials in $\left(x-x_{i}\right)$, for $r_{i}=1(1) 6$, and the sequence of operations for retrieving the coefficients $a_{i, m}$. For the sake of brevity we write, from now on, $a_{m}$ for $a_{i, m}$.

Denoting the coefficients of $\left(x-x_{i}\right)^{j}$ in $S_{i}(x)$ and $T_{i}(x)$ by $\alpha_{j}$ and $\beta_{j}$ respectively, the coefficients $a_{m}$ are retrieved as follows: For $r_{i}=1$ and 2, obvious. For $r_{i}=3, a_{0}=\alpha_{0} / \beta_{0}, a_{2}=\alpha_{1}-a_{0}, a_{1}=\beta_{0} / a_{2}$. For $r_{i}=4, a_{0}=\alpha_{0} / \beta_{0}, a_{1}=\beta_{0} /\left(\alpha_{1}-\right.$ $\left.a_{0} \beta_{1}\right), a_{3}=\beta_{1}-a_{1}, a_{2}=\left(\alpha_{1}-a_{0} \beta_{1}\right) / a_{3}$. For $r_{i}=5, a_{0}=\alpha_{0} / \beta_{0}, a_{1}=\beta_{0} /\left(\alpha_{1}-a_{0} \beta_{1}\right)$, $a_{2}=\left(\alpha_{1}-a_{0} \beta_{1}\right) /\left\{\beta_{1}-a_{1}\left(\alpha_{2}-a_{0}\right)\right\}, a_{4}=\alpha_{2}-a_{0}-a_{2}, a_{3}=\left\{\beta_{1}-a_{1}\left(\alpha_{2}-a_{0}\right)\right\}$ $/ a_{4}$. For $r_{i}=6, a_{0}=\alpha_{0} / \beta_{0}, a_{1}=\beta_{0} /\left(\alpha_{1}-a_{0} \beta_{1}\right), a_{2}=\left(\alpha_{1}-a_{0} \beta_{1}\right) /\left\{\beta_{1}-\right.$ $\left.a_{1}\left(\alpha_{2}-a_{0} \beta_{2}\right)\right\}, a_{3}=\left\{\beta_{1}-a_{1}\left(\alpha_{2}-a_{0} \beta_{2}\right)\right\} /\left\{\alpha_{2}-\left(a_{0}+a_{2}\right)\left(\beta_{2}-a_{1}\right)-a_{1} a_{0}\right\}, a_{5}=$ $\beta_{2}-a_{3}-a_{1}, a_{4}=\left\{\alpha_{2}-\left(a_{0}+a_{2}\right)\left(\beta_{2}-a_{1}\right)-a_{1} a_{0}\right\} / a_{5}$.

The retrieval process assumes that every $a_{m} \neq 0$ for $m \neq 0$. But, we may have $a_{0}=0$, and every retrieval step still goes through.

It seems likely from the above (verification left as an exercise for the reader) that for any $r_{i}$ there is a general procedure for retrieving $a_{0}, a_{1}, \cdots, a_{r_{i-3}}, a_{r_{i}-1}$, $a_{r_{i}-2}$, in that order, from the Euler-Minding formula [3], according to which $S_{i}(x)$ and $T_{i}(x)$ have these explicit expressions:

$$
\begin{aligned}
S_{i}(x)=a_{0} a_{1} \cdots & a_{r_{i}-1}\left(1+\sum_{j}^{0, r_{i}-2}\left(x-x_{i}\right) / a_{j} a_{j+1}\right. \\
+ & \sum_{j<k}^{0, r_{i}-3}\left(x-x_{i}\right)^{2} / a_{j} a_{j+1} a_{k+1} a_{k+2} \\
& \left.+\sum_{j<k<l}^{0, r_{i}-4} \sum\left(x-x_{i}\right)^{3} / a_{j} a_{j+1} a_{k+1} a_{k+2} a_{l+2} a_{l+3}+\cdots\right),
\end{aligned}
$$

and

$$
\begin{aligned}
T_{i}(x)=a_{1} a_{2} \cdots a_{r_{i}-1}(1+ & \sum_{j}^{1, r_{i}-2}\left(x-x_{i}\right) / a_{j} a_{j+1} \\
& \left.\quad+\sum_{j<k}^{1, r_{i}-3}\left(x-x_{i}\right)^{2} / a_{j} a_{j+1} a_{k+1} a_{k+2}+\cdots\right) .
\end{aligned}
$$

General Dynamics/Astronautics San Diego, California

1. L. M. Mrlne-Thomson, The Calculus of Finite Differences, Macmillan, London, 1933, Chapter V, p. 104-123.

2. P. I. Richards, Manual of Mathematical Physics, Pergamon, London and New York, 1959 , p. 257. p. $5-7$.

3. O. Perron, Die Lehre von den Kettenbrüchen, B. G. Teubner, Stuttgart, 1954, Vol. I, 